\title{
Improving the reliability of operation of electromechanical devices by means of ionistor-battery backup
}

\author{
W Biały ${ }^{1}, E M$ Stepanova $^{2}$, V I Zatsepina ${ }^{3}, E$ P Zatsepin ${ }^{3}$ and P I Skomorokhov ${ }^{4, *}$ \\ ${ }^{1}$ Department of Organization and Management of Silesian Technical University, Institute of Production Engineering, 26 Roosevelta \\ Street, Zabrze, 41-800, Poland \\ ${ }^{2}$ Department of Power Machinery of Kazan State Power Engineering University, 50 Krasnoselskaya Street, Kazan, 420066, Russia \\ ${ }^{3}$ Department of Electrical Equipment Lipetsk State Technical University, 30 Moskovskaya Street, Lipetsk, 398055, Russia \\ ${ }^{4}$ Electric Conditions Service the Branch of JSC "SO UPS" Regional Dispatch Control of Power Systems of the Lipetsk and Tambov \\ Regions, 20 "50 let NLMK” Street, Lipetsk, 398059, Russia
}

\begin{abstract}
This article is devoted to the problem of improving the operation reliability of electromechanical devices and devices at electric power industry facilities. Particular attention is paid to the fact that in the Russian power system electromechanical devices and devices designed for a voltage class of $110 \mathrm{kV}$ and higher are still the majority compared to microprocessor and microelectronic devices. Given their long service life and the inability to promptly replace all devices at once, improving the reliability of their operation is highly relevant. A schematic solution to the problem is developed. The scientific novelty of the proposed solution is of the fact that the redundant power supply circuit with operating current of electromechanical devices offers a consolidated use of innovative electric power storage devices, namely ionistors and classic rechargeable batteries combined according to a special circuit. This solution maximizes the operation reliability of both electromechanical devices and devices themselves, as well as their automatic control.
\end{abstract}

\section{Introduction}

Reliable operation of electromechanical devices is very important for the functioning of the unified energy system of Russia today [1-3]. One of the most significant factors is that the majority of relay protection and automation devices used in the power system of Russia in the voltage class of $110 \mathrm{kV}$ and above, has an electromechanical base. Making a quick transition to fully microprocessor-based protection and automation is very expensive for this voltage class.

Control systems of switches, disconnectors and other devices also have an electromechanical basis. As it is known, in $25-28 \%$ of cases, the cause of the largest system accidents that occurred in the world was relay protection failures [4]. It should be noted that the failure of the switching device or the failure of the output electromechanical relay can often occur due to the simple loss of the operating current or insufficient voltage to operate. Accordingly, to improve the reliability of the operation of electromechanical devices, it is necessary to provide the redundancy of their power supply [5-7].

\section{Formulation of the problem}

In order to reserve power and, as a result, increase the reliability of electromechanical devices and devices at a single power industry facility (substation, power station), the following solution is obvious: the installation of additional battery groups and the redundancy of DC circuits of electromechanical relays and switching device drives, that is, the usual backup of the local power supply system [8-9] However, this solution has significant drawbacks, the main of which is excessive high cost, because aside from additional batteries, a huge number of connecting wires for each electromechanical device will be required. In addition, the batteries are not able to give a quick discharge, if one needs a long simultaneous redundancy of a large number of electromechanical devices. Thus, the development of such a redundancy measure is required, so that any electromechanical device can have a power reserve sufficient both in power and in redundancy duration.

\section{Solution of the problem}

It is proposed to solve this problem by developing a method of consolidation of the advantages of the existing technical solutions in the field of ensuring the autonomous operation of electromechanical devices. As a part of the solution to this problem, particular attention should be paid to ionistors [10-11]. Their distinguishing features include a high specific capacity (of several farads), as well as an increased duration and reliability of the accumulated charge compared to similar devices.

*Corresponding author: pavelskomorokhov@mail.ru 
They are also distinguished by high reliability of operation in DC circuits with significant electromagnetic and network disturbances. The main advantages of using ionistors in comparison with classic batteries are: long service life; the ability to quickly charge and discharge; almost unlimited number of charge/discharge cycles; relatively low cost and no need to control the charging mode [12]. However, the use of only ionistors as a source of backup power supply for electromechanical devices is limited by the following disadvantages: low energy storage; low energy density; the need for serial connection of several tens or even hundreds of elements to achieve the required power of the accumulation of electricity.

According to the reference data, the ionistor stores energy equals to about $10 \%$ of the energy of a nickel metal hydride or lead battery. In this case, the battery produces a relatively constant operating voltage. It should be borne in mind that the electronic elements, which include the ionistor, operate at limited values of the supply voltage. The charge remaining in the ionistor when the minimum voltage is reached remains unused.

To ensure the redundant power supply of DC circuits of electromechanical devices (including significant electromagnetic and mains disturbances), it is proposed to use ionistors consolidated with classic lithium-ion (or similar) batteries through a specially designed circuit. In the proposed circuit solution, the ionistor will have to reduce the load on the battery, which significantly increases its service life, and at the same time increases the starting current that this installation is capable to give. In addition, the combined use of batteries with ionistors reduces the weight and dimensions of the first ones, which is important for saving space at the power industry facility.

First of all, it is necessary to determine the number of ionistors required to supply voltage to electromechanical devices of the electric power industry facility (taking into account the probability of complete termination of their power supply from the main source).First of all, it is necessary to calculate the amount of energy required to provide electromechanical devices with sufficient power $\mathrm{P}$ in the period of maximum consumption, which represents the failure of the main power source. The calculation must be made according to the following formula [13]:

$$
W_{i}=0.5 \cdot C_{i}\left(U_{\text {start }}^{2}-U_{\text {end }}^{2}\right),
$$

where $C_{i}$ is the total capacity of the ionistor group, F; $U_{\text {start }}$ is the voltage at the input of the ionistor group before the start of peak consumption, $\mathrm{V}$; $U_{\text {end }}^{2}$ is the voltage at the input of the ionistor group at the moment of the end of peak consumption, $\mathrm{V}$.

Taking into account the fact that a fully charged circuit is in a state of constant charging until the immediate start of operation, formula (1) will take the following form:

$$
W_{i}=0.5 \cdot C_{i} U_{n}^{2} .
$$

The best option for solving the task of ensuring redundancy are high-capacity ionistors with the following rating parameters: nominal voltage of a separate ionistor $U_{\mathrm{n}}=5.5 \mathrm{~V}$ and capacity $C=10 \mathrm{~F}$; permissible current of the charging and discharging of one ionistor is not more than 1.2 A. Based on statistical and experimental data, ionistors of this type have a mean time to failure equal to 15,000 cycles, with a service life of at least 12 years [14].

In order to efficiently store the electric power required for this consolidated scheme, it is necessary to limit the charge voltage of the ionistor to a level slightly lower than its allowable voltage. It is further proposed to calculate the required number of ionistor based on the following formula:

$$
N_{i}=\frac{U_{n}}{U_{i}} .
$$

The total stock of energy and power of the ionistor group will be determined on the basis of reference data using the following formulas:

$$
\left\{\begin{array}{l}
W_{i . \text { sum }}=C \cdot \frac{U^{2}}{2} \cdot N_{i} ; \\
P_{i . \text { sum }}=\frac{U^{2}}{4 R} \cdot N_{i},
\end{array}\right.
$$

where $C$ is the capacity of a separate ionistor, $\mathrm{F}$; $U$ is the voltage at the input terminals of an electromechanical device, $\mathrm{V} ; R$ is the effective series resistance.

On the basis of calculations performed using formulas (1) - (4) the type of ionistors according to the reference books is accepted. The conditions of their operations is consolidated with classic batteries, that's why it is necessary to ensure compliance of the total capacities of ionistors and batteries. For the previously selected parameters and the number of ionistors, we take three batteries with a nominal capacity of 3744 Ah each as batteries. Figure 1 shows the developed circuit solution for power redundancy of the operating current circuits of electromechanical devices and apparatuses.

The installation consists of $N_{i}$ parallel connected ionistors with selected parameters, which are connected to three parallel connected batteries. The direct current circuit of the reserved electromechanical apparatus operates as the load $R_{\text {load }}$. Note that the developed connection scheme is universal.

The developed solution has a wide range of applications at power facilities. For example, during operation of the automatic circuit reclosers (ACRs), there is a possibility of failure of the ACR device because of insufficient operation voltage to the intermediate or output relay [15]. To exclude such failures, it is proposed to apply the developed redundancy scheme as follows (Figure 2). The red solid lines in Figure 2 depict the integration of the developed circuit design, while the red dashed lines indicate the possibility of an additional separate redundant power 


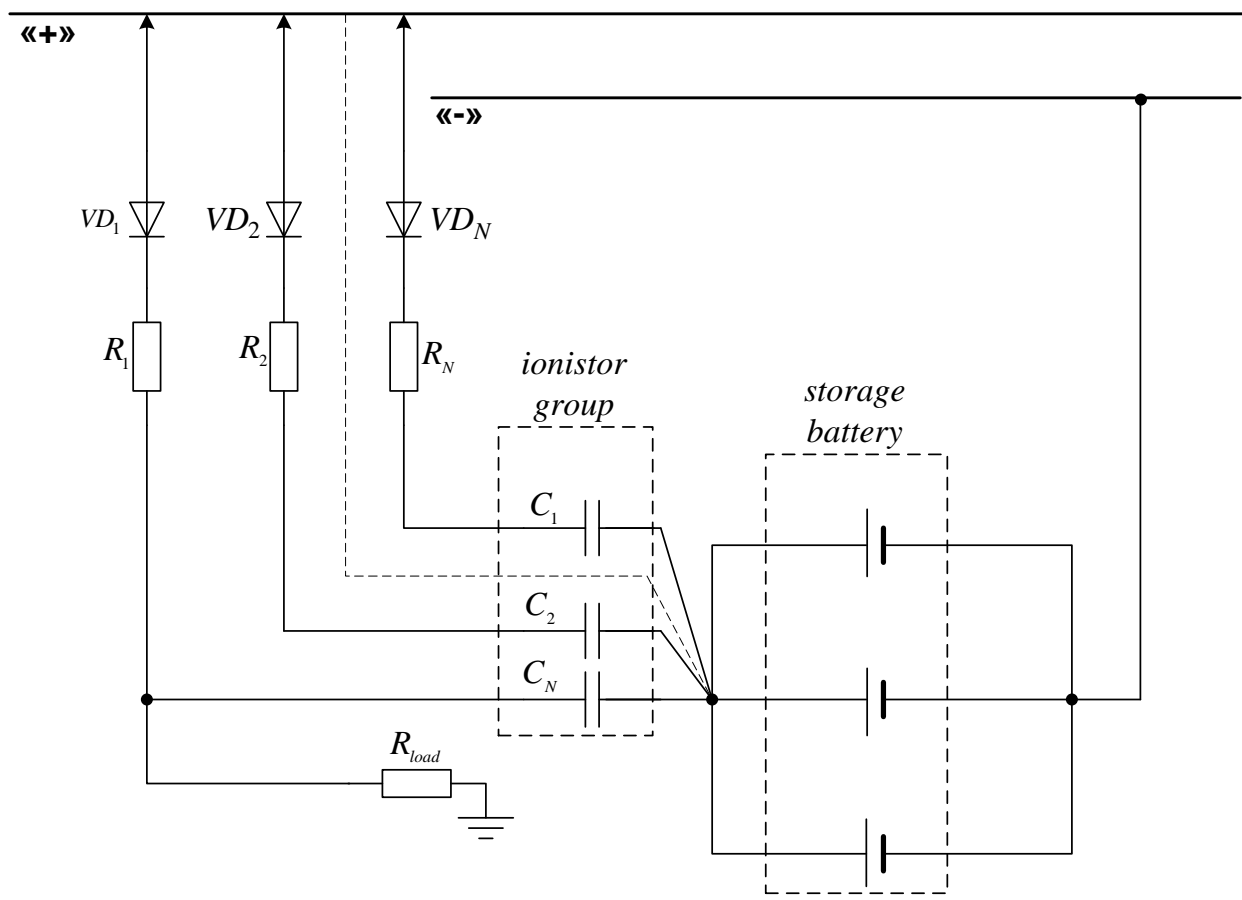

Fig. 1. Redundant DC power supply circuit for electromechanical devices.

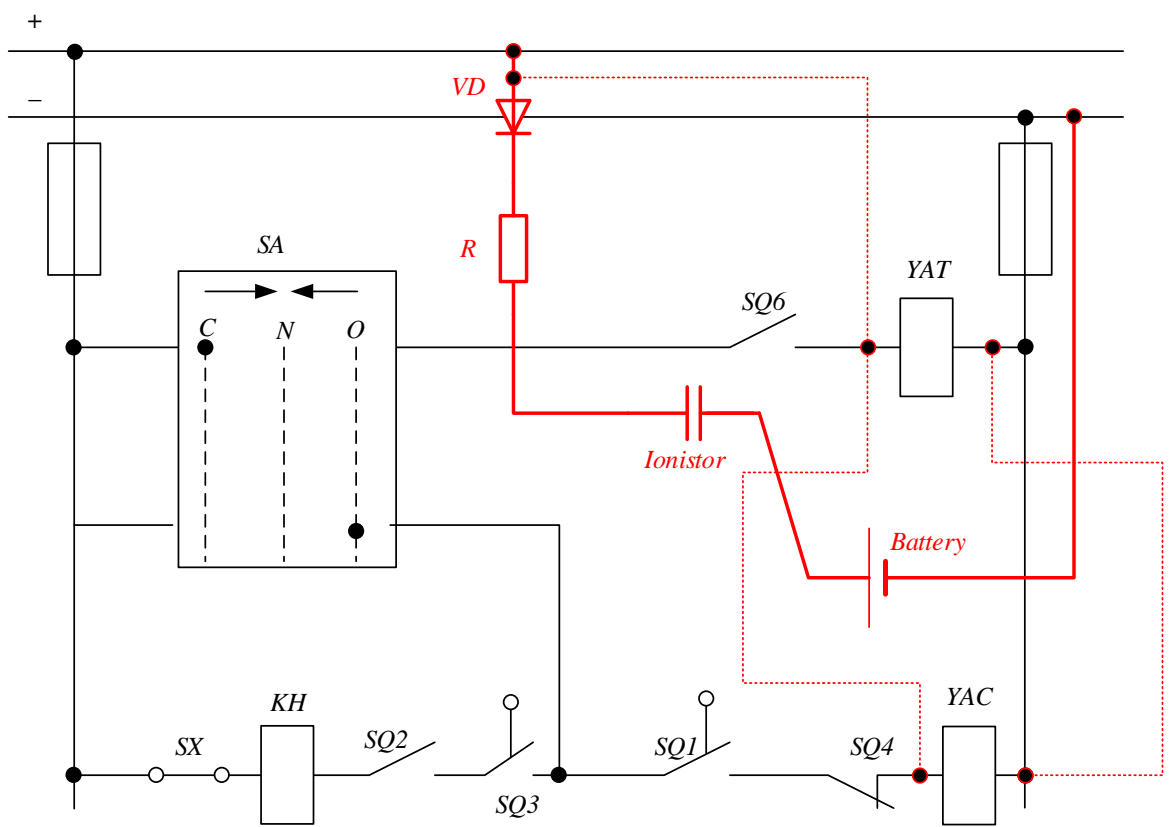

Fig. 2. Integration of the developed circuit design into the AR circuit: $S A$ - reclosure mode selection key; $Y A T$ shutdown solenoid; $Y A C$ - switching electromagnet; $K H$ - voltage relay; $S X$ - voltage control pad; $S Q 1-S Q 4$, SQ6 block of intermediate relay contacts.

supply of the electromagnet on (YAC) and off (YAT) while installing an additional auxiliary contacts in the mounted circuit.

\section{Conclusion}

The proposed circuit solution has the following advantages:
- Allows to reserve several electromechanical devices on DC circuits at once for a long time for a period, which is usually sufficient to eliminate even serious failures and resume normal operation of DC systems of the electric power industry object;

- Combines the advantages of ionistors and classic rechargeable batteries as a source of backup power for electromechanical devices, leveling the main disadvantages of both separate methods; 
- Increases the reliability of electromechanical devices and devices in general through structural redundancy, by introducing a backup innovative battery into the system, as well as reducing the number of failures in relay protection and automation devices based on electromechanical base;

- Has a versatility of installation. It can be used also in AC circuits of electromechanical devices and apparatuses. In this case, it is necessary to provide the installation of an inverting device at the output of the circuit.

\section{References}

1. N.V. Savina, Y.V. Myasoedov, L.A. Myasoedova, Influence of quality of the electric energy on reliability of electrical supply systems, Int. multiconf. on industrial engineering and modern technologies (Vladivostok), 1-5 (2018)

2. A.N. Shpiganovich, V.I. Zatsepina, A.A. Shpiganovich, V.M. Stepanov, Power-supply systems reliability control, EAI endorsed transactions on energy web, 5(19), 155083 (2018)

3. P. Wang, J.Q. Xu, J. Su, The research of urban distribution network high-reliability power supply construction, International Conf. on Advanced Power System Automation and Protection, 1497$1500(2011)$

4. O.O. Akhmedova, Error analysis of relay protection systems in the energy sector, Energy and Resource Saving: Industry and Transport, 3, 19-22 (2016)

5. V.I. Zatsepina, E.P. Zatsepin, Reliability of power supply systems under the influence of negative factors, Int. Conf. on Industrial Engineering, Application and Manufacturing, 1-4 (2017)

6. L. Janak, Z. Hadas, J. Smilek, Reliability assessment of electromechanical energy harvesting systems establishment of devices key characteristics for application in safety-critical systems, 17th Int. Conf. on Mechatronics - Mechatronika, 1-4 (2016)

7. C. Yang, Q. Liu, T. Li, The reliability model of Micro-electromechanical system based on fracture mechanism, The Proceedings of 9th Int. Conf. on Reliability, Maintainability and Safety, 378-382 (2011)

8. E.P. Zatsepin, Informational and measuring system for EAF, Stal', 3, 23-27 (2004)

9. V.V. Vdovin, D.P. Visloguzov, V.A. Klan, D.A. Kotin, V.V. Pankratov, A.V. Smetannikov, DC mains backup power system for frequencycontrolled electric drive, 15th Int. Conf. of Young Specialists on Micro/nanotechnologies and Electron devices, 387-391 (2014)

10. V. Kuznetsov, O. Pan'kina, N. Machkovskaya, Electric double layer capacitors (ionistors): design and production, Components and Technologies, 50, 12-16 (2005)

11. A.A. Filimonova, E.P. Zatsepin, V.I. Zatsepina, Multilevel control or power consumption at metallurgical plants, Int. Russian Automation Conf., 1-4 (2018)
12. V.I. Grits, T.V. Dubovoy, I.Ya. Shestakov, Ionistors, Actual Problems of Aviation and Astronautics, 1(11), 240-241 (20015)

13. H. Yang, Analysis of supercapacitor charge redistribution through constant power experiments, IEEE Power \& Energy Society General Meeting, 15 (2017)

14. Super capacitors: 2002-2012. Available at: http://www.electrosad.ru/Electronics/SuperCon.htm (Accessed 30 September 2018).

15. A. Edwards, R. Heerden, S. Chowdhury, S.P. Chowdury, H. Kang, Fast auto-reclose function for $765 \mathrm{kV}$ lines in the proximity of resonant line voltages, 10th IET Int. Conf. on Developments in Power System Protection. Managing the Change, 14 (2010) 